\title{
छु \\ Electronic and geometric structure of the PTCDA/Ag(110) interface probed by angle-resolved photoemission
}

\author{
M. Wießner, D. Hauschild, A. Schöll, and F. Reinert \\ Universität Würzburg, Experimentelle Physik VII and Röntgen Research Center for Complex Material Systems RCCM, 97074 Würzburg, \\ Germany and Karlsruher Institut für Technologie KIT, Gemeinschaftslabor für Nanoanalytik, 76021 Karlsruhe, Germany \\ V. Feyer \\ Peter Grünberg Institute (PGI-6), JARA-FIT, Research Center Jülich, 52425 Jülich, Germany and NanoESCA beamline, Sincrotrone Trieste, \\ in Area Science Park, 34149 Basovizza, Trieste, Italy
}

\author{
K. Winkler and B. Krömker \\ Omicron NanoTechnology GmbH, Limburger Strasse 75, 65232 Taunusstein, Germany
}

(Received 30 May 2012; published 11 July 2012)

\begin{abstract}
The properties of molecular films are determined by the geometric structure of the first layers near the interface. These are in contact with the substrate and feel the effect of the interfacial bonding, which particularly, for metal substrates, can be substantial. For the model system 3,4,9,10-perylenetetracarboxylic dianhydride on $\mathrm{Ag}(110)$, the geometric structure of the first monolayer can be modified by preparation parameters. This leads to significant differences in the electronic structure of the first layer. Here, we show that, by combining angle-resolved photoelectron spectroscopy with low-energy electron diffraction, we cannot only determine the electronic structure of the interfacial layer and the unit cell of the adsorbate superstructure, but also the arrangement of the molecules in the unit cell. Moreover, in bilayer films, we can distinguish the first from the second layer and, thus, study the formation of the second layer and its influence on the buried interface.
\end{abstract}

DOI: 10.1103/PhysRevB.86.045417

PACS number(s): 33.60.+q, 61.05.jh, 73.20.Hb, 79.60.Dp

\section{INTRODUCTION}

The interface to the substrate determines the properties of molecular films and is, thus, crucial for electronic devices based on these materials. In the case of metal contacts, the metal-molecule interface is decisive for the charge injection or extraction, and in various papers, it has been shown that the geometric structure of the interfacial layer influences the electronic structure in the vicinity of the interface ${ }^{1,2}$ and the (epitaxial) growth beyond the first monolayer. ${ }^{3-6}$ However, experimental access to the microscopic arrangement of molecules is not straightforward and, in most cases, is performed by a combination of low-energy electron diffraction (LEED) and scanning tunneling microscopy (STM). ${ }^{7-9}$ Moreover, LEED-IV (Refs. 10-12) or photoelectron diffraction ${ }^{13-15}$ (PED) can also be applied. Although these approaches have provided valuable information on various organic adsorbate systems on the (sub)monolayer level, their potential is limited when trying to determine the registry between successive layers and to analyze buried layers. In addition, LEED-IV and PED demand complicated modeling and scattering calculations. In this article, we show that a combination of LEED and angle-resolved photoelectron spectroscopy (ARPES) can provide access to the electronic and geometric structures of molecular layers. On the example of the well-known model system, 3,4,9,10-perylenetetracarboxylic dianhydride ${ }^{16-25}$ (PTCDA) on a $\operatorname{Ag}(110)$ surface, we demonstrate that ARPES can complement diffraction data and can provide the arrangement of the molecules in the unit cell determined by LEED. Moreover, we show, on bilayer systems, that this approach is not limited to monolayer systems. We find that the PTCDA molecules originally built a brick-wall first layer after deposition, whereas, annealing of multilayer films leads to a herringbone monolayer. The different arrangements can be distinguished in LEED and can lead to different photoemission spectra and angular-intensity distributions in ARPES. In the case of bilayer samples, the second layer grows in a herringbone arrangement and leaves the brick-wall order of the first layer unchanged and shows no significant influence on the signature of the interfacial layer in the photoemission spectra.

\section{EXPERIMENTAL DETAILS}

All experiments were performed in an ultrahigh vacuum system with a base pressure below $10^{-10}$ mbar. The PTCDA films were prepared in an attached preparation chamber by organic molecular-beam deposition with a deposition rate of $0.2 \mathrm{ML} / \mathrm{min}$ onto clean and well-ordered $\mathrm{Ag}(110)$ singlecrystal surfaces ${ }^{26}$ at room temperature.

The angle-integrated photoemission measurements were obtained at room temperature with a monochromatized UV lamp for $\mathrm{He}_{\alpha}$ radiation $(h v=21.2 \mathrm{eV})$ and a photoelectron analyzer (Scienta SES200), leading to an overall energy resolution of about $\Delta E_{\text {inst }}=10 \mathrm{meV}$.

For the parallel momentum $k_{x, y}$-resolved photoemission intensity distributions, we used the NanoESCA photoemission spectrometer (FOCUS GmbH/Omicron Nanotechnology $\mathrm{GmbH}){ }^{27-29}$ The setup includes a nonmagnetic electrostatic photoelectron emission microscope and an aberration compensated double-pass hemispherical analyzer. This instrument is equipped with a transfer lens behind the immersion lens objective to map the angular distribution of the photoelectrons by imaging the focal plane. In $k$-imaging mode, the NanoESCA ${ }^{27}$ resolves the complete hemisphere of the emitted photoelectrons with a $k$ resolution of about $0.05 \AA^{-1}$. The energy resolu- 
tion for our current paper was set to about $\Delta E_{\mathrm{inst}}=200 \mathrm{meV}$, which is sufficient for the investigated system and facilitates sufficient transmission. The measurement times, with this method, shrink dramatically down to minutes compared to measurements taken for many hours using conventional angleresolving photoelectron spectrometers. For the measurements, we used both unmonochromatized $\mathrm{He}$ I radiation in the laboratory and synchrotron radiation with $h v=55 \mathrm{eV}$ at the NanoESCA beamline, ${ }^{30}$ a branch of the Nanospectroscopy beamline $1.2 \mathrm{~L}$ at Elettra (Trieste) $24 .{ }^{31}$ Checks for radiation damage were performed by monitoring the valence band, and no spectral changes were observed after $20 \mathrm{~min}$; this time was sufficient for the measurement with acquisition times of 5-10 min. The polarization effect on the momentum-dependent photoemission intensity distribution was reduced by using the $C_{2}$ and $\sigma_{y z}$ symmetry operation on the monolayer data sets and the $C_{2}$ symmetry operation on the multilayer data set, thus, respecting the symmetry of the substrate and of the organic layer that is already apparent on the raw data level.

\section{RESULTS AND DISCUSSIONS}

Figure 1 displays the LEED diffractograms of two different PTCDA monolayers on $\operatorname{Ag}(110)$. Figure 1(a) shows the well-known commensurate phase with a superstructure matrix given by $\left(\begin{array}{cc}2 & 3 \\ -2 & 3\end{array}\right)$, which is obtained directly by depositing 1 ML PTCDA on the substrate at room temperature. ${ }^{17,32}$ STM investigations showed that this LEED pattern corresponds to molecules arranged in a brick-wall phase. ${ }^{17,33}$ Polymorphism in the bulk structure is a general observation for this class of molecular materials, which are mainly bonded by van der Waals and electrostatic forces. The occurrence of various geometric structures is also common for ultrathin epitaxial films as long as the adsorbate-substrate bond is not too strong, and several findings demonstrate that it can be influenced by the preparation condition. Figure 1(b) shows the LEED diffractogram of 1 ML PTCDA, which was prepared by evaporating about three layers of PTCDA and consequent annealing at $T=550 \mathrm{~K}$ for $5 \mathrm{~min}$. At this temperature, the multilayer molecules desorb leaving the stronger bonded first monolayer on the substrate. ${ }^{34}$ It is striking that the geometric structure of the monolayer clearly differs from the brick-wall phase in Fig. 1(a). This
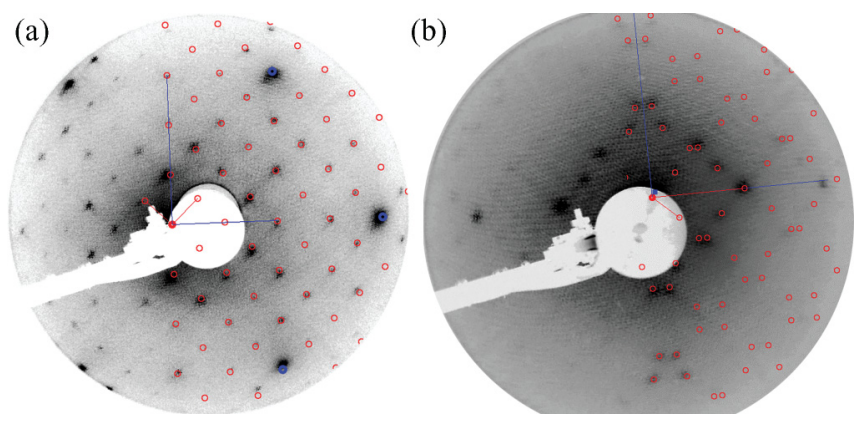

FIG. 1. (Color online) LEED diffractograms of one monolayer PTCDA on $\mathrm{Ag}(110)$. On the right side, the respective diffractograms are superposed by a simulation of the substrate (blue) and adsorbate spots (red). (a) shows the commensurate brick-wall phase recorded with $82 \mathrm{eV}$ electron energy, whereas, (b) shows the herringbone phase at $36 \mathrm{eV}$ electron energy.

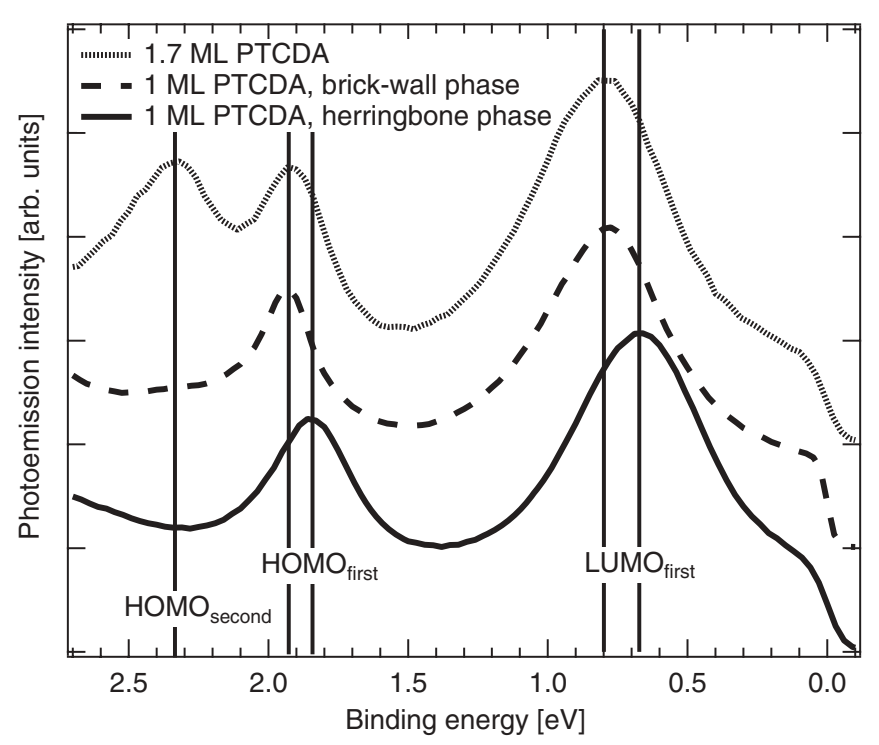

FIG. 2. Photoemission spectra taken with $h v=21.2 \mathrm{eV}$ at $k_{x}=0, k_{y}=1.7 \AA^{-1}$ of the valence regime of PTCDA/Ag(110) in the herringbone (solid curve) and brick-wall monolayer phases (dashed curve) as well as of a bilayer film (dotted curve, coverage 1.7 ML) normalized on the lowest unoccupied molecular orbital (LUMO) intensity. The prominent signals of the monolayers are denoted by $\mathrm{LUMO}_{\text {first }}$ and highest occupied molecular orbital $\left(\mathrm{HOMO}_{\text {first }}\right)$, and for the second layer, the prominent signals are denoted by $\mathrm{HOMO}_{\text {second }}$.

adsorption phase has already been reported by Seidel et al., ${ }^{35}$ and a herringbone arrangement was identified by STM. However, our analysis of the LEED pattern derives a slightly different superstructure matrix given by $\left(\begin{array}{cc}0 & -11 \\ 1.9 & 3\end{array}\right) \pm 0.2$.

The unit-cell vectors of the adsorbate are, thus, $a=31.8$ and $b=11.6 \AA$, with an enclosed angle of $42^{\circ}$. The resulting unit-cell area is $(245 \pm 30) \AA^{2}$, providing space for two molecules, but it is smaller than twice the area of the brick-wall structure $\left(141.4 \AA^{2}\right) .{ }^{17,32}$ The adsorbate phase derived by annealing has approximately the same unit-cell area as the well-known herringbone phase of PTCDA on the $\operatorname{Ag}(111)$ surface (238.7 $\AA^{2}$ ). Moreover, also on $\mathrm{Ag}(100)$, PTCDA grows in a herringbone phase. There, the unit cell $\left(267 \AA^{2}\right)$ of the first monolayer is significantly larger, which is induced by the particular substrate geometry, but the second monolayer again shows the preferred dense packing with a unit-cell size of $\left(242 \AA^{2}\right){ }^{36}$ In the following, the two distinct monolayer phases of PTCDA/Ag(110) will be utilized to demonstrate the capabilities of ARPES in resolving the differences in the electronic and geometric structures. Figure 2 shows the photoemission spectra of one monolayer of PTCDA/Ag(110) in the brick wall (dashed curve) and herringbone phases (solid curve) as well as for a bilayer film with a coverage of 1.7 ML (dotted curve). The spectra were recorded at an emission direction tilted by $55^{\circ}$ against the surface normal in the [110] direction, thus, resulting in $k_{x}=0$ and $k_{y}=1.7 \AA^{-1}$. The signal closest to the Fermi level $\left(E_{B}=0 \mathrm{eV}\right)$ of the substrate can be assigned to the lowest unoccupied molecular orbital of the PTCDA molecule, which is occupied in the monolayer by charge transfer from the substrate 21,37 and is 
labeled as $\mathrm{LUMO}_{\text {first }}$ in the following. At a binding energy of approximately $E_{B}=1.9 \mathrm{eV}$, the former highest occupied molecular orbital of PTCDA in the first monolayer is observed (denominated as $\mathrm{HOMO}_{\text {first }}$ ). Upon adsorption of more than one monolayer, the HOMO of the second layer appears at $E_{B}=2.3 \mathrm{eV}$ (denoted as $\mathrm{HOMO}_{\text {second }}$ ). The energy positions of the peak maxima are indicated by vertical lines in Fig. 2. Between the brick-wall and the herringbone phases, a clear energy shift occurs for the $\mathrm{LUMO}_{\text {first }}$ and $\mathrm{HOMO}_{\text {first }}$, which can be quantified to $\approx 120$ and $\approx 100 \mathrm{meV}$, respectively. In accordance with similar observations for PTCDA/Ag(111) (Refs. 38 and 39), we associate this energy shift with a weaker bonding of the PTCDA to the $\operatorname{Ag}(110)$ substrate in the case of the herringbone phase. This most probably goes along with a longer vertical bonding distance as compared to the bonding distance of $2.59 \AA$ of the perylene backbone in the brick-wall phase. ${ }^{24}$ Additionally, the lateral film order changes, which may contribute to the energy shift in the photoemission spectra. ${ }^{40}$ Moreover, the occurrence of two instead of one adsorption site in the case of the herringbone phase ${ }^{38}$ explains the larger linewidths in the respective spectra. The full width at half maximum of the $\mathrm{HOMO}_{\text {first }}$, e.g., increases from the commensurate brick-wall phase with $220-300 \mathrm{meV}$ in the herringbone phase. In order to investigate the effect of the different molecular orientations in the unit cell, the angledependent photoemission intensity distribution measurements of the $\mathrm{HOMO}_{\text {first }}$ and $\mathrm{LUMO}_{\text {first }}$ are displayed in Fig. 3. The photoemission intensity distributions can be related to the wave function of the respective molecular orbital. ${ }^{41-43}$ For PTCDA, these $k$ maps show very characteristic differences between the $\mathrm{HOMO}_{\text {first }}$ and the $\mathrm{LUMO}_{\text {first }}{ }^{37,44}$ Figures 3(b) and 3(c) show the $\mathrm{LUMO}_{\text {first }}$ and the $\mathrm{HOMO}_{\text {first }}$ intensity distributions of the brick-wall phase measured with the NanoESCA and $55 \mathrm{eV}$ photons. The theoretically calculated intensity distribution is indicated by the dotted isointensity lines in each graph. These data reproduce the findings of Ziroff et al ${ }^{37}$ and Puschnig et al. ${ }^{44}$ The intensity at the $\Gamma$ point as reported in Ref. 37 does not appear in our data probably due to a different measurement geometry and a larger photon energy of $55 \mathrm{eV}$. The latter leads to a much larger $k_{z}$ value so that the hybridization state is substantially suppressed. If we turn to the herringbone phase in Figs. 3(e) and 3(f), the photoemission pattern changes dramatically. The $\mathrm{LUMO}_{\text {first }}$ now shows four high-intensity regions in contrast to the two prominent maxima of the brickwall structure. A similar occurrence happens to the $\mathrm{HOMO}_{\text {first }}$ where the photoemission also shows a doubling of all features. This is obvious since the two molecules in the unit cell of the herringbone phase are rotated by $90^{\circ}$ against each other, causing the additional photoemission intensities. Identical findings have been made for several photon energies in the range from 25 to $60 \mathrm{eV}$ excluding a photon-energy-caused doubling of the features with respect to the brick-wall phase. Together with the LEED results, we can derive real-space models of the two monolayer phases, which are shown in Figs. 3(a) and 3(d). For the herringbone phase, Fig. 3(d) shows the only possible configuration of molecules for the LEED-derived unit cell where the molecules do not touch each other within their van der Waals radii. An alternative scenario with two differently rotated domains of molecules with only one molecular orientation can be excluded by the LEED. Our real-space
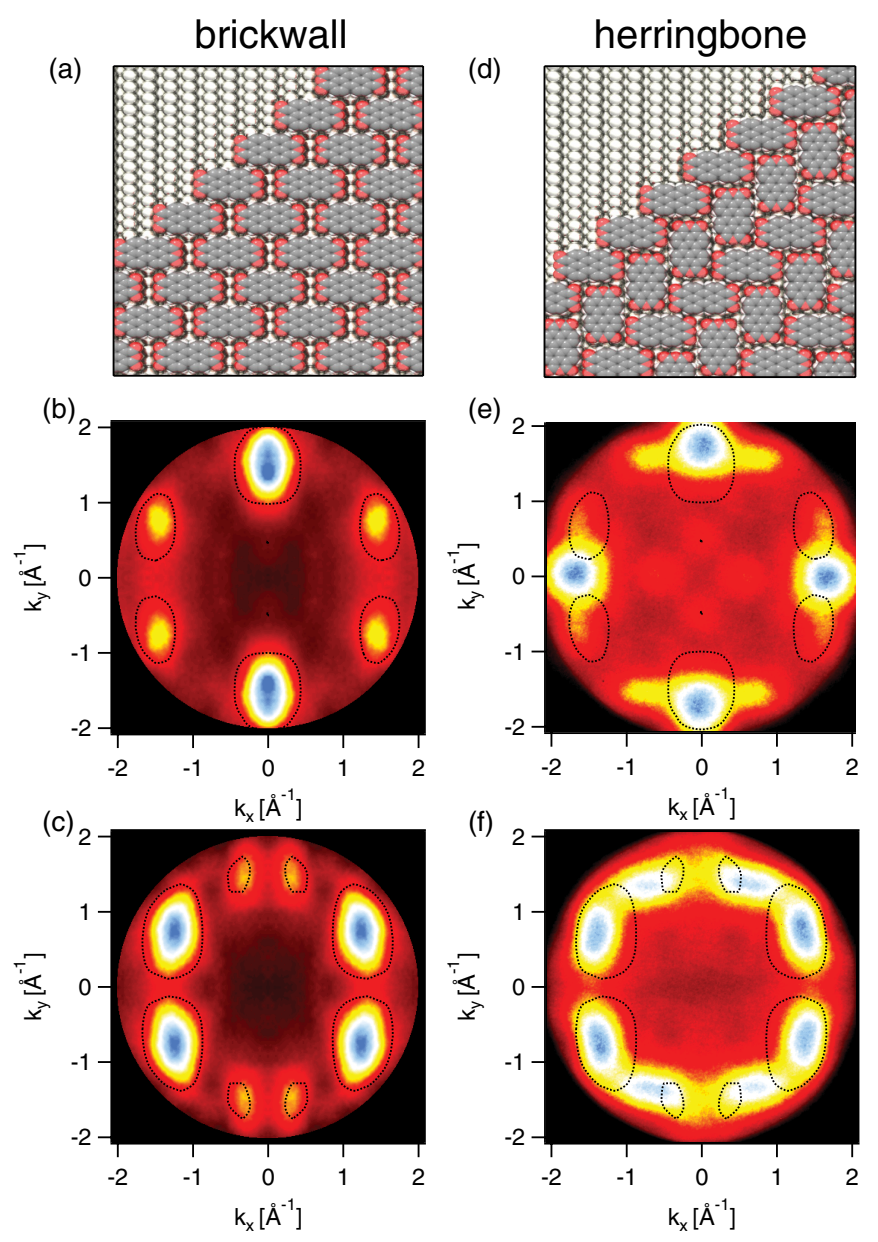

FIG. 3. (Color online) Momentum-dependent photoemission intensity distributions of one ML PTCDA on $\mathrm{Ag}(110)$. Panel (a) shows the real-space model derived from the results of the LEED and ARPES measurements for the brick-wall phase. Panel (b) shows the photoemission intensity distribution for the $\mathrm{LUMO}_{\text {first }}$, and panel (c) shows the photoemission intensity distribution for the $\mathrm{HOMO}_{\text {first }}$ of the brick-wall phase using $55 \mathrm{eV}$ photons. (d)-(f) show the real-space model, the $\mathrm{LUMO}_{\text {first }}$ and the $\mathrm{HOMO}_{\text {first }}$ of the herringbone phase recorded with $22.1 \mathrm{eV}$ photons. The dotted circles in the $k$ maps represent isointensity lines of the theoretical intensity distribution for an isolated PTCDA molecule reproduced from Ref. 37.

models, thus, corroborate the STM measurements by Seidel et al. ${ }^{32}$

On the basis of the understanding of the ARPES and LEED data of the PTCDA monolayers, in the following, we will investigate the more complex PTCDA bilayer. The dotted curve in Fig. 2 shows the photoemission spectra of a PTCDA film on $\operatorname{Ag}(110)$ with a coverage of about 1.7 ML. In addition to the spectral features of the brick-wall monolayer, the bilayer spectrum shows some intensity on the higherbinding energy side of the $\mathrm{HOMO}_{\text {first }}$. In accordance with the findings on similar systems, ${ }^{16,21}$ this signal is attributed to the $\mathrm{HOMO}_{\text {second }}$ of the second layer. The momentum-dependent photoemission intensities for the 1.7 ML PTCDA sample are shown in Fig. 4. Figure 4(a) displays the $\mathrm{LUMO}_{\text {first }}$, which is absent in pure multilayer spectra ${ }^{21}$ and can, therefore, be related to the first monolayer. From Fig. 4(a), one can 

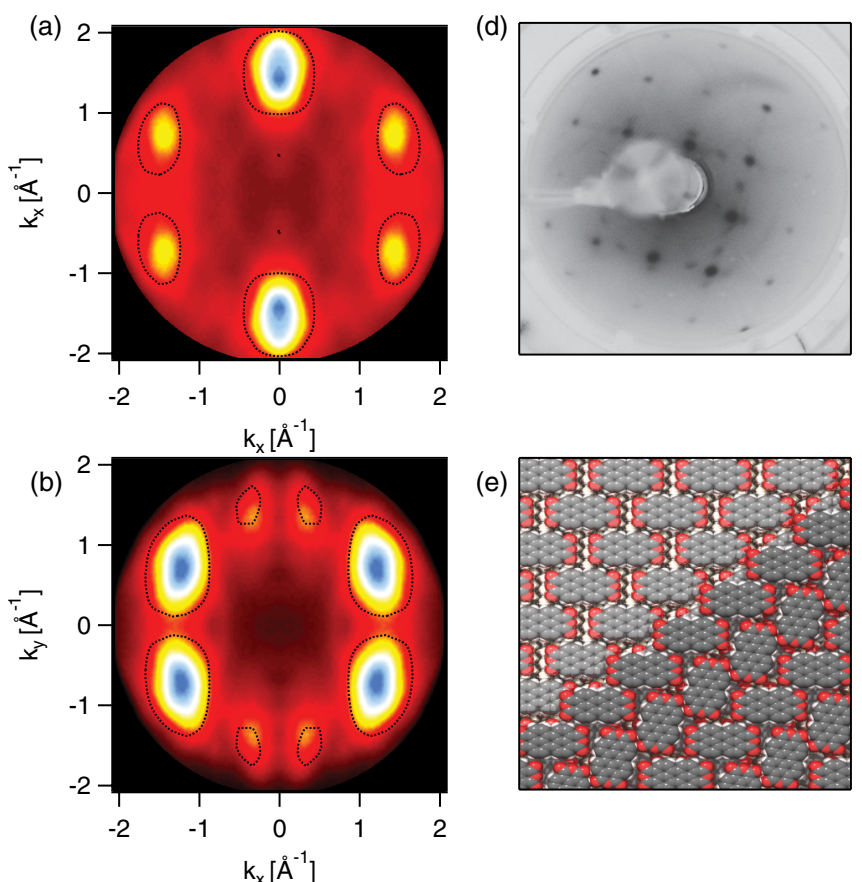

(e)
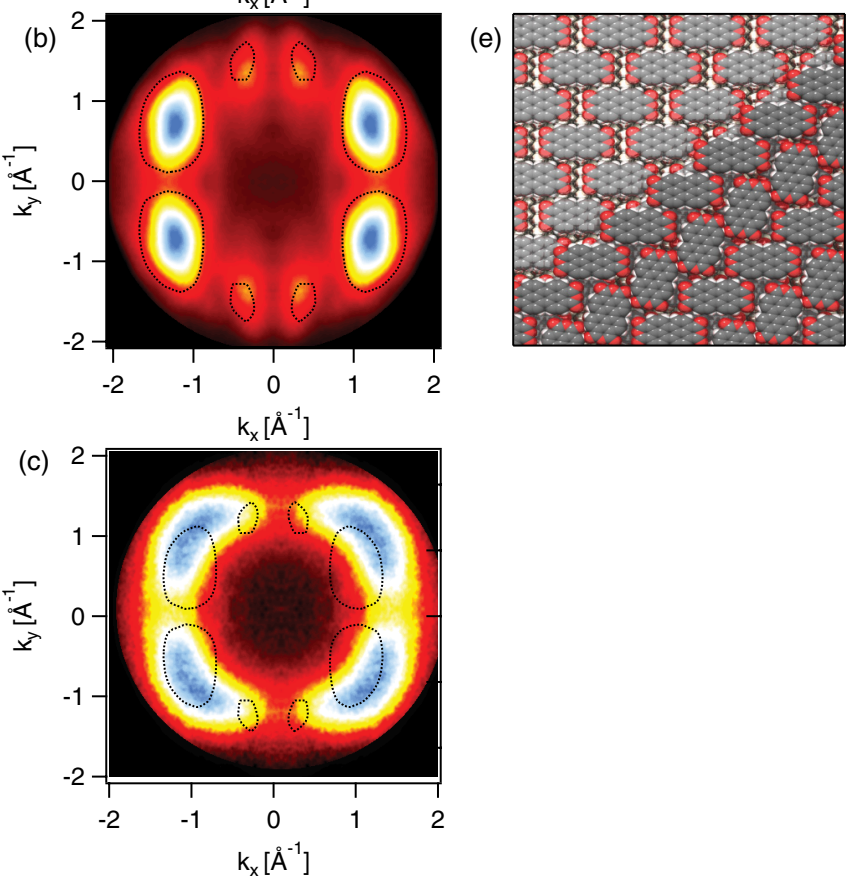

FIG. 4. (Color online) PTCDA bilayer (coverage $\approx 1.7 \mathrm{ML}$ ) on $\mathrm{Ag}(110)$ : Momentum-dependent photoemission intensity distribution recorded with $55 \mathrm{eV}$ photons at the binding energy of the (a) $\mathrm{LUMO}_{\text {first }}$, of the (b) $\mathrm{HOMO}_{\text {first }}$, and at the (c) $\mathrm{HOMO}_{\text {second }}$ (after the $\mathrm{HOMO}_{\text {first }}$ intensity was subtracted). (d) LEED diffractogram of the bilayer recorded with $15 \mathrm{eV}$ electron energy. (e) The resulting real-space model of the PTCDA bilayer with a brick wall first and a herringbone second layer.

immediately follow that the interfacial layer remains in the brick-wall configuration upon additional PTCDA deposition. This is corroborated by Fig. 4(b), which shows the HOMO first, also matching the $\mathrm{HOMO}_{\text {first }}$ of the brick-wall monolayer in Fig. 3(c). Figure 4(c) is the angular-intensity distribution of the $\mathrm{HOMO}_{\text {second }}$ and shows clear similarity with the $\mathrm{HOMO}_{\text {first }}$ of the herringbone monolayer in Fig. 3(f). However, on closer inspection, differences occur, which can be explained by a relative rotation of the molecules in the second layer unit cell, which differs from the $90^{\circ}$ in the monolayer herringbone phase. A best fit of the experimental data was derived for a superposition of two $\mathrm{HOMO}_{\text {first }}$ maps with a relative rotation angle of $100^{\circ}$, a similar angle as can be found in the $\alpha$ phase of bulk PTCDA. ${ }^{45,46}$

The LEED diffractogram in Fig. 4(d) supports the ARPES findings. It shows the contribution from the brick-wall monolayer by the characteristic quadratically arranged spots and additional weaker spots in the same positions as for the herringbone monolayer in Fig. 1(b). Thus, we conclude that the first layer is still in the brick-wall arrangement. Moreover, a comparison of the photoemission spectra of the brick-wall monolayer and the bilayer in Fig. 2 shows that no significant modification of the electronic structure of the first layer occurs upon adsorption of the second layer PTCDA. The second layer itself grows in a herringbone configuration. A possible real-space model of the bilayer is shown in Fig. 4(e), showing one mirror domain of the second layer.

\section{CONCLUSION}

To summarize, we have demonstrated that combining lowenergy electron diffraction with angle-resolved photoelectron spectroscopy is a very powerful method to elucidate the geometric and electronic structures of ordered molecular adsorbates. We applied this approach to the two different monolayer phases of PTCDA on $\operatorname{Ag}(110)$ and found, in agreement with literature, ${ }^{17,35}$ that directly after deposition, the molecules grow in a commensurate superstructure with a brick-wall arrangement of the molecules along the [001] axis of the substrate. Annealing of multilayer films results in a monolayer with a different point-on-line superstructure with two molecules in a herringbone configuration in the unit cell. Moreover, the interfacial bonding obviously differs between the two monolayer phases, resulting in different energy positions of the molecular frontier orbitals. Upon deposition of more than one layer, the molecules start to form the second layer in a herringbone configuration. The buried interfacial layer can be clearly identified in LEED and ARPES even for bilayer samples. We find that the additional second layer leaves the brick-wall arrangement in the first layer intact and does not significantly change the electronic structure.

\section{ACKNOWLEDGMENTS}

We gratefully thank C. Wiemann and C. M. Schneider for their cooperation at the NanoESCA at Elettra and acknowledge Elettra for providing good quality synchrotron light. We thank P. Puschnig (Graz) for stimulating discussions. This work was financially supported by the Deutsche Forschungsgemeinschaft (Grants No. FOR 1162 and No. GRK 1221) and the Bundesministerium für Bildung und Forschung (Contracts No. 05K10WW2 and No. 03SF0356B).
${ }^{1}$ S. Duhm, G. Heimel, I. Salzmann, H. Glowatzki, R. L. Johnson, A. Vollmer, J. P. Rabe, and N. Koch, Nature Mater. 7, 326 (2008).
${ }^{2}$ W. Chen, S. Chen, S. Chen, Y. L. Huang, H. Huang, D. C. Qi, X. Y. Gao, J. Ma, and A. T. S. Wee, J. Appl. Phys. 106, 064910 (2009). 
${ }^{3}$ S. R. Forrest, Chem. Rev. 97, 1793 (1997).

${ }^{4}$ E. Umbach, K. Glöckler, and M. Sokolowski, Surf. Sci. 402-404, 20 (1998).

${ }^{5}$ Q. Chen, T. Rada, A. McDowall, and N. V. Richardson, Chem. Mater. 14, 743 (2002).

${ }^{6}$ M. Eremtchenko, J. Schaefer, and F. Tautz, Nature (London) 425, 602 (2003).

${ }^{7}$ C. Ludwig, B. Gompf, J. Petersen, R. Strohmaier, and W. Eisenmenger, Z. Phys. B: Condens. Matter 93, 365 (1994).

${ }^{8}$ F. Sellam, T. Schmitz-Hübsch, M. Toerker, S. Mannsfeld, H. Proehl, T. Fritz, K. Leo, C. Simpson, and K. Müllen, Surf. Sci. 478, 113 (2001).

${ }^{9}$ Q. Chen, A. McDowall, and N. Richardson, Langmuir 19, 10164 (2003).

${ }^{10}$ M. A. Van Hove, R. Lin, and G. A. Somorjai, Phys. Rev. Lett. 51, 778 (1983).

${ }^{11}$ G. Michalk, W. Moritz, H. Pfnür, and D. Menzel, Surf. Sci. 129, 92 (1983).

${ }^{12}$ G. Held and D. Menzel, Phys. Rev. Lett. 74, 4221 (1995).

${ }^{13}$ J. Osterwalder, T. Greber, A. Stuck, and L. Schlapbach, Phys. Rev. B 44, 13764 (1991).

${ }^{14}$ Applications of Synchrotron Radiation: High-Resolution Studies of Molecules and Molecular Adsorbates on Surfaces, 1st ed., edited by W. Eberhardt et al., Structure Determination of Molecular Adsorbates Using Photoelectron Diffraction, Springer Series in Surface Sciences (Springer, Berlin, 1994), pp. 127-169.

${ }^{15}$ D. Woodruff, Surf. Sci. Rep. 62, 1 (2007).

${ }^{16}$ M. Jung, U. Baston, G. Schnitzler, M. Kaiser, J. Papst, T. Porwol, H. Freund, and E. Umbach, J. Mol. Struct. 293, 239 (1993).

${ }^{17}$ K. Glöckler, C. Seidel, A. Soukopp, M. Sokolowski, E. Umbach, M. Böhringer, R. Berndt, and W.-D. Schneider, Surf. Sci. 405, 1 (1998).

${ }^{18}$ B. Krause, A. C. Dürr, K. Ritley, F. Schreiber, H. Dosch, and D. Smilgies, Phys. Rev. B 66, 235404 (2002).

${ }^{19}$ F. Schreiber, Phys. Status Solidi A 201, 1037 (2004).

${ }^{20}$ A. Hauschild, K. Karki, B. C. C. Cowie, M. Rohlfing, F. S. Tautz, and M. Sokolowski, Phys. Rev. Lett. 94, 036106 (2005).

${ }^{21}$ Y. Zou, L. Kilian, A. Scholl, T. Schmidt, R. Fink, and E. Umbach, Surf. Sci. 600, 1240 (2006).

${ }^{22}$ F. S. Tautz, Prog. Surf. Sci. 82, 479 (2007).

${ }^{23}$ L. Romaner, D. Nabok, P. Puschnig, E. Zojer, and C. AmbroschDraxl, New J. Phys. 11, 053010 (2009).

${ }^{24}$ A. Abbasi and R. Scholz, J. Phys. Chem. C 113, 19897 (2009).

${ }^{25}$ C. Weiss, C. Wagner, R. Temirov, and F. S. Tautz, J. Am. Chem. Soc. 132, 11864 (2010).
${ }^{26}$ G. Nicolay, F. Reinert, S. Schmidt, D. Ehm, P. Steiner, and S. Hüfner, Phys. Rev. B 62, 1631 (2000).

${ }^{27}$ B. Krömker, M. Escher, D. Funnemann, D. Hartung, H. Engelhard, and J. Kirschner, Rev. Sci. Instrum. 79, 053702 (2008).

${ }^{28}$ C. Mathieu, N. Barrett, J. Rault, Y. Y. Mi, B. Zhang, W. A. de Heer, C. Berger, E. H. Conrad, and O. Renault, Phys. Rev. B 83, 235436 (2011).

${ }^{29}$ C. Wiemann, M. Patt, I. P. Krug, N. B. Weber, M. Escher, M. Merkel, and C. M. Schneider, e-J. Surf. Sci. Nanotechnol. 9, 395 (2011).

${ }^{30} \mathrm{http}: / / \mathrm{www}$. elettra.trieste.it/elettra-beamlines/nanoesca.html.

${ }^{31}$ A. Locatelli, A. Bianco, D. Cocco, S. Cherifi, S. Heun, M. Marsi, M. Pasqualetto, and E. Bauer, J. Phys. IV (France) 104, 99 (2003).

${ }^{32}$ C. Seidel, C. Awater, X. Liu, R. Ellerbrake, and H. Fuchs, Surf. Sci. 371, 123 (1997).

${ }^{33}$ M. Böhringer, W.-D. Schneider, K. Glöckler, E. Umbach, and R. Berndt, Surf. Sci. 419, L95 (1998).

${ }^{34}$ L. Kilian, E. Umbach, and M. Sokolowski, Surf. Sci. 573, 359 (2004).

${ }^{35}$ C. Seidel, J. Poppensieker, and H. Fuchs, Surf. Sci. 408, 223 (1998).

${ }^{36}$ J. Ikonomov, O. Bauer, and M. Sokolowski, Surf. Sci. 602, 2061 (2008).

${ }^{37}$ J. Ziroff, F. Forster, A. Schöll, P. Puschnig, and F. Reinert, Phys. Rev. Lett. 104, 233004 (2010).

${ }^{38}$ L. Kilian, A. Hauschild, R. Temirov, S. Soubatch, A. Schöll, A. Bendounan, F. Reinert, T.-L. Lee, F. S. Tautz, M. Sokolowski, and E. Umbach, Phys. Rev. Lett. 100, 136103 (2008).

${ }^{39}$ A. Hauschild, R. Temirov, S. Soubatch, O. Bauer, A. Schöll, B. C. C. Cowie, T.-L. Lee, F. S. Tautz, and M. Sokolowski, Phys. Rev. B 81, 125432 (2010).

${ }^{40}$ S. Soubatch, C. Weiss, R. Temirov, and F. S. Tautz, Phys. Rev. Lett. 102, 177405 (2009).

${ }^{41}$ S. Kera, S. Tanaka, H. Yamane, D. Yoshimura, K. Okudaira, K. Seki, and N. Ueno, Chem. Phys. 325, 113 (2006).

${ }^{42}$ G. Koller, S. Berkebile, M. Oehzelt, P. Puschnig, C. AmbroschDraxl, F. P. Netzer, and M. G. Ramsey, Science 317, 351 (2007).

${ }^{43}$ P. Puschnig, S. Berkebile, A. J. Fleming, G. Koller, K. Emtsev, T. Seyller, J. D. Riley, C. Ambrosch-Draxl, F. P. Netzer, and M. G. Ramsey, Science 326, 702 (2009).

${ }^{44}$ P. Puschnig, E.-M. Reinisch, T. Ules, G. Koller, S. Soubatch, M. Ostler, L. Romaner, F. S. Tautz, C. Ambrosch-Draxl, and M. G. Ramsey, Phys. Rev. B 84, 235427 (2011).

${ }^{45}$ S. R. Forrest, M. L. Kaplan, and P. H. Schmidt, J. Appl. Phys. 55, 1492 (1984).

${ }^{46}$ M. Möbus, N. Karl, and T. Kobayashi, J. Cryst. Growth 116, 495 (1992). 